\title{
Rottlerin induces cyclooxygenase-2 upregulation through an ATF4 and reactive oxygen species-independent pathway in HEI-OC1 cells
}

\author{
SEON MIN WOO ${ }^{1 *}$, WOO KEUN LEE ${ }^{2 *}$, KYOUNG-JIN MIN ${ }^{1}$, \\ DONG EUN KIM ${ }^{2}$, SOON HYUNG PARK ${ }^{2}$, SUNG IL NAM ${ }^{2}$ and TAEG KYU KWON ${ }^{1}$ \\ Departments of ${ }^{1}$ Immunology and ${ }^{2}$ Otolaryngology, School of Medicine, \\ Keimyung University, Daegu 700712, Republic of Korea
}

Received June 30, 2015; Accepted April 29, 2016

DOI: $10.3892 / \mathrm{mmr} .2016 .5320$

\begin{abstract}
Hearing loss can be caused by infection, inflammation, loud noise and ototoxic drugs. The induction of cyclooxygenase-2 (COX-2) expression is an important event during the cellular inflammatory response. The present study investigated the effect of rottlerin on COX-2 mRNA and protein expression in HEI-OC1 cells. Cell viability was determined using an MTT assay. Western blotting was used to examine the expression of COX-2, endoplasmic reticulum stress-associated transcription factors and activation of the MAPK pathway. ROS was measured using the fluorescent probe 2', 7'-dichlorodihydrofluorescein diacetate. Treatment with the natural protein kinase $\mathrm{C} \delta$ inhibitor, rottlerin, was shown to increase COX-2 expression at the protein and mRNA levels in a dose-dependent manner. Rottlerin was shown to induce increased reactive oxygen species (ROS) generation, however, ROS were not critical for rottlerin-induced upregulation of COX-2 expression in HEI-OC1 cells. In addition, rottlerin was shown to increase the phosphorylation of p38 mitogen-activated protein kinase (MAPK). The pharmacological inhibition of p38MAPK and suppression of activating transcription factor 4 (an ER stress-associated transcription factor) expression by small interfering RNA inhibited rottlerin-induced COX-2 upregulation. Furthermore, COX-2 expression levels were increased
\end{abstract}

Correspondence to: Dr Sung Il Nam, Department of Otolaryngology, School of Medicine, Keimyung University, 194 DongSan-Dong, Jung-Gu, Daegu 700712, Republic of Korea E-mail: entnamsi@dsmc.or.kr

Dr Taeg Kyu Kwon, Department of Immunology, School of Medicine, Keimyung University, 194 DongSan-Dong, Jung-Gu, Daegu 700712, Republic of Korea

E-mail:kwontk@dsmc.or.kr

${ }^{*}$ Contributed equally

Key words: rottlerin, cyclooxygenase-2, activating transcription factor 4, p38 mitogen-activated protein kinase, HEI-OC1 cells further when cells were treated with rottlerin and interleukin-1 $\beta$ or protein kinase $\mathrm{C}$ activator, PMA. In conclusion, the results of the present study demonstrated that rottlerin is a novel inducer of COX-2 expression and identified the mechanisms involved in this process. Rottlerin may be considered a potential activator of repair and remodeling.

\section{Introduction}

Ototoxicity is the tendency of a drug or chemical agent to cause inner ear dysfunction, producing symptoms of hearing loss and/or dizziness. Auditory cells can be injured by infection, inflammation, loud noise and ototoxic drugs. The inflammatory response to injury must be accompanied by tissue repair, remodeling, induction of repair and remodeling capacity may inhibit ototoxicity.

Prostaglandin-endoperoxide synthases, also termed cyclooxygenases $(\mathrm{COX})$, are the rate-limiting enzymes that catalyze the production of prostanoids from arachidonic acid. COX enzymes are categorized into two subtypes, COX-1 and COX-2. COX-2 expression is induced during inflammatory processes (1-3). A previous study demonstrated that auditory cells produce prostaglandin E2 (PGE2) in response to lipopolysaccharide (LPS) stimulation via increased COX2 expression. PGE2 production may be involved in tissue repair and remodeling in the organ of Corti (4).

Rottlerin is a pigmented plant compound isolated from the gland hair covering the fruit of Mallotus philippensis. Gschwendt et al (5) and other studies have demonstrated that rottlerin is a specific inhibitor of protein kinase $\mathrm{C}(\mathrm{PKC}) \delta(6-8)$. However, certain previous studies observed that rottlerin has a variety of PKC 8 -independent actions (9-12). The current study aimed to investigate whether rottlerin affects the gene expression levels of COX-2. The present study demonstrated that rottlerin-induced COX-2 upregulation is associated with the activation of p38 mitogen-activated protein kinase (MAPK) and increased expression of activating transcription factor (ATF) 4, an endoplasmic reticulum (ER) stress-associated transcription factor. Furthermore, the ability of rottlerin to induce COX-2 expression was independent of reactive oxygen species (ROS) generation. 


\section{Materials and methods}

Cells and materials. Mouse auditory HEI-OC1 cells were purchased from the House Ear Institute (Los Angeles, CA, USA) and cultured in sodium pyruvate-free Dulbecco's modified Eagle's medium (Gibco; Thermo Fisher Scientific, Inc., Waltham, MA, USA) supplemented with $10 \%$ heat-inactivated fetal bovine serum, $100 \mathrm{U} / \mathrm{ml}$ penicillin and $100 \mathrm{mg} / \mathrm{ml} \mathrm{strep-}$ tomycin (all obtained from Welgene, Inc., Gyeongsan, Korea) at $37^{\circ} \mathrm{C}$ in a humidified $5 \% \mathrm{CO}_{2}$ atmosphere. Recombinant interleukin (IL)- $1 \beta$ was purchased from R\&D Systems, Inc. Minneapolis, MN, USA). Phorbol-12-myristate-13-acetate (PMA) was obtained from EMD Millipore (Billerica, MA, USA). Rottlerin, PD98059, SB203580 and SP600125 were purchased from Enzo Life Sciences, Inc. (Farmingdale, NY, USA). Glutathione (GSH), N-acetylcysteine (NAC) and trolox were purchased from Sigma-Aldrich (St. Louis, MO, USA). Polyclonal mouse anti-COX-2 antibody (1:1,000, cat. no. 160106) was obtained from the Cayman Chemical Company (Ann Arbor, MI, USA). Polyclonal rabbit anti-p46/54 c-Jun N-terminal kinase (JNK, 1:700, cat. no. 9252), polyclonal rabbit anti-phospho (p)-p46/54 JNK (1:700, cat. no. 9251), polyclonal rabbit anti-p38MAPK (1:700, cat. no. 9211), polyclonal rabbit anti-p-p38MAPK (1:700, cat. no. 9212), polyclonal rabbit anti-extracellular signal-regulated kinase (ERK1/2, 1:700, cat. no. 9102) and polyclonal rabbit anti-p-ERK1/2 (1:700, cat. no. 9101) antibodies were obtained from Cell Signaling Technology, Inc. (Danvers, MA, USA). Polyclonal rabbit anti-ATF4 (1:1,000, cat. no. sc-200) and polyclonal rabbit anti-ATF3 (1:700, cat. no. sc-188) antibodies were purchased from Santa Cruz Biotechnology, Inc. (Dallas, TX, USA). Polyclonal rabbit anti-regulated in development and DNA damage responses 1 (REDD1) antibody (1:1,000, cat. no. 10638-1-AP) was obtained from ProteinTech Group, Inc. (Chicago, IL, USA). Monoclonal mouse anti-actin antibody (1:15,000, cat. no. A5441) was purchased from Sigma-Aldrich.

Western blot analysis. For the western blotting, cells were washed with cold phosphate-buffered saline (PBS) and lysed on ice in modified radioimmunoprecipitation assay buffer (50 mM Tris- $\mathrm{HCl} \mathrm{pH} \mathrm{7.4,} 1 \%$ NP-40, $0.25 \%$ Na-deoxycholate, $150 \mathrm{mM} \mathrm{NaCl}, 1 \mathrm{mM} \mathrm{Na} \mathrm{VO}_{4}$ and $1 \mathrm{mM} \mathrm{NaF}$ ) containing protease inhibitors (100 $\mu \mathrm{M}$ phenylmethylsulfonyl fluoride, $10 \mu \mathrm{g} / \mathrm{ml}$ leupeptin, $10 \mu \mathrm{g} / \mathrm{ml}$ pepstatin, and $2 \mathrm{mM}$ EDTA). The lysates were centrifuged at $10,000 \mathrm{xg}$ for $10 \mathrm{~min}$ at $4^{\circ} \mathrm{C}$ and the supernatant fractions were collected. The protein concentration was determined using Micro BCA protein assay kit was from Pierce Chemical (Rockford, IL, USA), according to the manufacturer's protocol. An equal amount of cell lysate $(60 \mu \mathrm{g})$ was dissolved in sample buffer, and samples were boiled for $5 \mathrm{~min}$. The proteins were separated by $10 \%$ sodium dodecyl sulfate-polyacrylamide gel electrophoresis and transferred to Immobilon-P membranes (Amersham, Uppsala, Sweden) and were subsequently blocked for $1 \mathrm{~h}$ at room temperature in $5 \%(\mathrm{w} / \mathrm{v})$ non-fat dried milk, and then were incubated for overnight at room temperature with the aforementioned primary antibodies, followed by an incubation with biotinylated secondary antibodies. The specific proteins were detected using an enhanced chemiluminescence western blotting kit (Merck Millipore, Darmstadt, Germany) according to the manufacturer's instructions. The band intensity of COX-2 protein was measured using Image $\mathbf{J}$ (National Institutes of Health, Bethesda, MA, USA), and expressed as a ratio to actin band intensity.

Reverse transcription-polymerase chain reaction (RT-PCR). Total RNA was isolated using TRIzol reagent (Invitrogen; Thermo Fisher Scientific, Inc.) and $1 \mu \mathrm{g}$ of total RNA was used to synthesize cDNA using M-MLV RT (Gibco; Thermo Fisher Scientific, Inc.) according to the manufacturer's instructions. Then, $2 \mu \mathrm{l}$ of cDNA was amplified with Taq (Solgent, GeNetBio, South Korea) kits in a total volume of $25 \mu 1$ according to the manufacturer's protocol. The PCR primers were purchased from Macrogen (Seoul, South Korea). The following primers were used for amplification: Sense 5'-ACA CACTCTATCACTGGCACC-3' and antisense 5'-TTCAGG GAGAAGCGTTTGC-3' for COX-2; and sense 5'-ACGACA TGGAGAAGATCTGG-3' and antisense 5'-TCAGGCAGC TCATAGCTCTT-3' for actin. The PCR amplification was performed using the following cycling conditions: $94^{\circ} \mathrm{C}$ for $3 \mathrm{~min}$; 17 (actin) or 25 cycles (COX-2) of $94^{\circ} \mathrm{C}$ for $45 \mathrm{sec}, 58^{\circ} \mathrm{C}$ for $45 \mathrm{sec}, 72^{\circ} \mathrm{C}$ for $1 \mathrm{~min}$; and a final extension step at $72^{\circ} \mathrm{C}$ for $10 \mathrm{~min}$. The amplified products were separated by electrophoresis on a 1.5\% agarose gel (Invitrogen; Thermo Fisher Scientific, Inc.) and detected under UV light.

Cell viability assay. MTT assay was used to determine the cell viability using WelCount Cell Viability Assay kit (WelGENE, South Korea). Briefly, $24 \mathrm{~h}$ after drug treatment the reagent was added to each well and then measured with multi-well plate reader (at $450 / 690 \mathrm{~nm}$ ).

Transfection and small interfering RNA (siRNA). HEI-OC1 cells were seeded at a density of $1 \times 10^{5}$ cells/well in 6-well culture plates one day prior to transfection to achieve 50-60\% confluency. The ATF4 and green fluorescent protein (GFP) control siRNA duplexes used in the present study were purchased from Santa Cruz Biotechnology, Inc. and Invitrogen; Thermo Fisher Scientific, Inc., respectively. Cells were transfected with siRNA oligonucleotides using Oligofectamine reagent (Invitrogen; Thermo Fisher Scientific, Inc.) according to the manufacturer's protocol.

Measurement of ROS. Intracellular accumulation of ROS was determined using the fluorescent probe 2', 7'-dichlorodihydrofluorescein diacetate (H2DCFDA). $\mathrm{H}_{2}$ DCFDA is commonly used to measure ROS generation (13). Cells were seeded in a 6 -well plate for $24 \mathrm{~h}$ prior to treatment with $5 \mu \mathrm{M}$ rottlerin, and treated with $5 \mu \mathrm{M}$ H2DCFDA for $30 \mathrm{~min}$. Cells were then trypsinized (Welgene, Inc., Gyeongsan, South Korea) and resuspended in PBS. Fluorescence was measured at the desired time intervals using a flow cytometer (BD FACSCanto II; BD Biosciences, Franklin Lakes, NJ, USA) or detected using a fluorescence microscope (Axiovert200; Zeiss GmbH, Jena, Germany).

Statistical analysis. Values are expressed as the mean \pm standard deviation of at least three independent experiments. Data were analyzed with one-way analysis of variance and Student-Newman-Keuls post hoc comparisons using the 
Statistical Package for Social Sciences software version 22.0 (IBM SPSS, Armonk, NY, USA). P<0.05 was considered to indicate a statistically significant difference.

\section{Results}

Rottlerin induces COX-2 expression in HEI-OCl cells. To determine whether rottlerin affects the mRNA and protein expression levels of COX-2, HEI-OC1 cells were treated with various rottlerin concentrations $(0.5-5.0 \mu \mathrm{M})$ for $12 \mathrm{~h}$. As demonstrated in Fig. 1A, COX-2 protein levels were increased by rottlerin in a dose-dependent manner. To elucidate the correlation between COX-2 protein and COX-2 mRNA in HEI-OC1 cells, RT-PCR was performed. The levels of COX-2 mRNA were also increased by rottlerin in a dose-dependent manner. Additionally, the cytotoxic effects of rottlerin were evaluated by MTT assay. Cell viability following rottlerin treatment was determined to be $>90 \%$ using the MTT assay (Fig. 1C).

ROS generation did not affect rottlerin-induced COX-2 expression in HEI-OCl cells. Several previous studies have suggested that ROS are associated with signaling mechanisms that induce COX-2 expression $(14,15)$. Therefore, the present study examined whether rottlerin induces ROS production using H2DCFDA-derived fluorescence and fluorescence-activated cells sorting detection in HEI-OC1 cells. As presented in Fig. 2A and B, compared with untreated cells, rottlerin significantly increased the intracellular ROS levels within $10 \mathrm{~min}$ of treatment $(\mathrm{P}<0.0001)$. Additionally, the present study investigated whether blockade of rottlerin-induced ROS generation affects COX-2 expression levels in HEI-OC1 cells. Compared with rottlerin treatment, pretreatment with the antioxidants NAC, GSH and trolox did not inhibit rottlerin-induced COX-2 protein expression (Fig. 2C). These results suggest that ROS are not involved in rottlerin-induced COX-2 expression.

Rottlerin-induced COX-2 expression is associated with activation of $38 M A P K$. The current study investigated the effect of rottlerin on MAPK activity in order to determine whether this signaling pathway is involved in rottlerin-induced COX-2 expression. As shown in Fig. 3A, rottlerin markedly increased the phosphorylation of p38MAPK, but not of ERK and JNK. p38MAPK activation was detected at $3 \mathrm{~h}$ and subsequently increased in a time-dependent manner (Fig. 3A). To determine the importance of individual MAPK pathways in rottlerin-induced COX-2 expression, the present study examined the effects of PD98059 (a potent ERK inhibitor), SB203580 (a specific p38MAPK inhibitor) and SP600125 (a potent JNK inhibitor) on rottlerin-induced COX-2 expression. As presented in Fig. 3B, pretreatment with SB203580 in the presence of rottlerin resulted in a 0.57 -fold decrease in COX-2 protein expression levels compared with rottlerin treatment, but SP600125 and PD98059 did not affect COX-2 protein expression. To further investigate the correlation between the p38MAPK pathway and rottlerin-induced COX-2 expression, SB203580 dose-response experiments were conducted in rottlerin-treated HEI-OC1 cells. As demonstrated in Fig. 3C, SB203580 markedly reduced
A

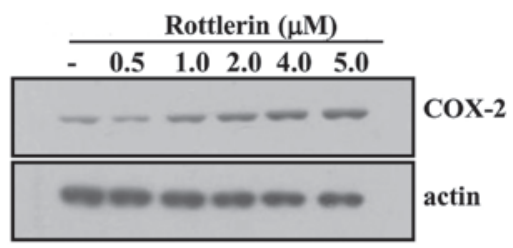

B

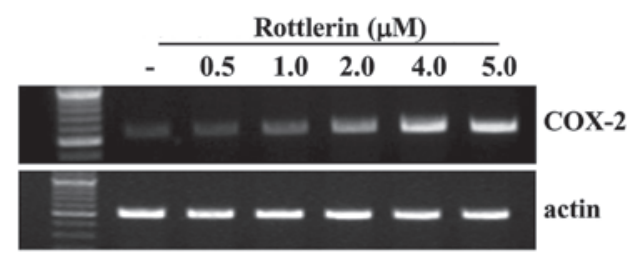

C

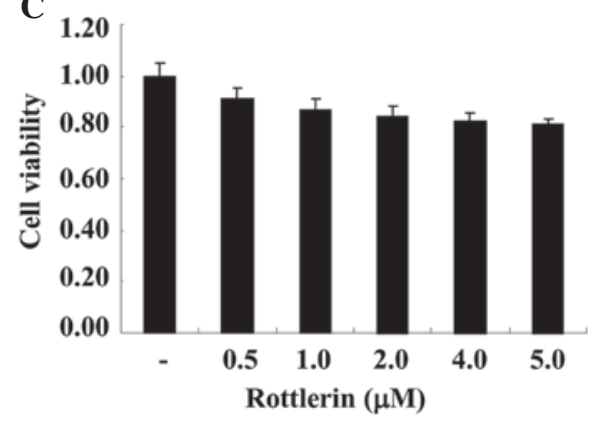

Figure 1. Rottlerin increased the expression levels of COX-2 in HEI-OC1 cells. HEI-OC1 cells were incubated with the indicated concentrations of rottlerin for $12 \mathrm{~h}$. (A) Protein expression levels of COX-2 and actin were determined by western blotting. (B) mRNA expression levels of COX-2 and actin were determined by RT-PCR. The level of actin was used as a loading control for western blot and RT-PCR analysis. (C) HEI-OC1 cells were incubated with the indicated concentrations with rottlerin for $24 \mathrm{~h}$. Cell viability was examined by MTT reduction assays and the results are expressed as the percentage of surviving cells relative to control cells. The cell viability was not significantly different between the Rottlerin-treated and the control cells. COX-2 cyclooxygenase-2. RT-PCR, reverse transcription-polymerase chain reaction.

COX-2 protein expression levels in a dose-dependent manner. Together these results indicate that the activation of p38MAPK pathway is important for the regulation of rottlerin-induced COX-2 expression in HEI-OC1 cells.

Rottlerin induces COX-2 expression via activation of the ATF4 transcription factor. A previous study demonstrated that ER stress-inducing agents can induce COX-2 expression (16). Therefore, kinetic studies were performed on rottlerin-treated HEI-OC1 cells to determine the effects of ER stress-associated transcription factors (REDD, ATF 3 and 4) on rottlerin-induced COX-2 expression. Incubation with rottlerin caused a time-dependent increase in COX-2 protein expression (Fig. 4). Notably, ATF4 protein expression levels were markedly increased by treatment with rottlerin compared with untreated cells. However, ATF3 and REDD1 levels were not identified to be increased in response to rottlerin (Fig. 4A). To examine whether rottlerin-induced COX-2 expression is dependent on ATF4 activity, an siRNA duplex targeting AFT4 mRNA was used. HEI-OC1 cells transfected with the control (GFP) or ATF4 siRNA were treated with rottlerin for $12 \mathrm{~h}$. Suppression of ATF4 expression by 


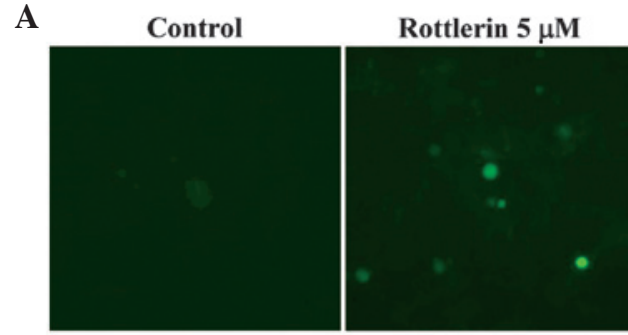

B

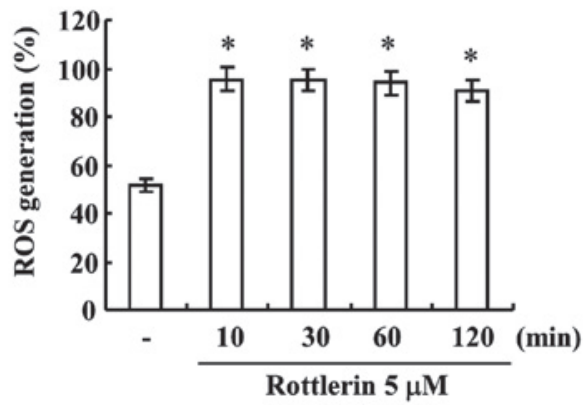

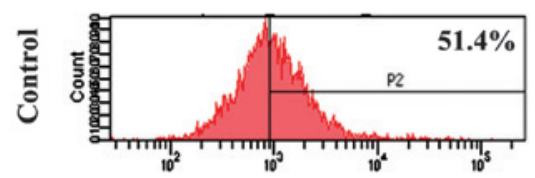

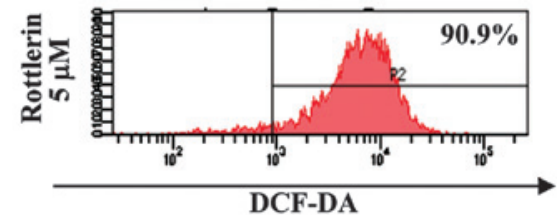

$\mathbf{C}$

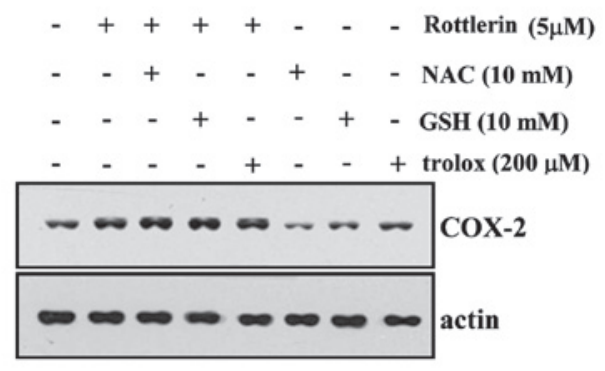

Figure 2. Effect of ROS generation on COX-2 expression in rottlerin treated HEI-OC1 cells. HEI-OC1 cells were treated with $5 \mu \mathrm{M}$ rottlerin for $10,30,60$ and $120 \mathrm{~min}$, and then loaded with a DCF-DA fluorescent dye. DCF-DA fluorescence intensity was detected by (A) fluorescence microscopy (left panel) and flow cytometry (right panel) at $2 \mathrm{~h}$. Magnificatio, $\mathrm{x} 200$. (B) Rottlerin $(5 \mu \mathrm{M})$ increased ROS generation following 10, 20, 60 and 120 min treatment, as measured by flow cytometry. (C) HEI-OC1 cells were pretreated with $10 \mathrm{mM} \mathrm{NAC,} 10 \mathrm{mM} \mathrm{GSH}$ and $200 \mu \mathrm{M}$ trolox, and then $5 \mu \mathrm{M}$ rottlerin for a further $12 \mathrm{~h}$. The protein expression levels of COX-2 and actin were determined by western blotting. The level of actin was used as a loading control. "P<0.001 vs. control. DCF-DA, dichloro-dihydro-fluorescein diacetate; ROS, reactive oxygen species; NAC, N-acetylcysteine; GSH, glutathione; COX-2, cyclooxygenase-2.

transfection with siRNA inhibited rottlerin-induced upregulation of COX-2 protein levels compared with control siRNA (Fig. 4B). These results suggest that the expression of ATF4 is critical for rottlerin-induced COX-2 expression.

Rottlerin enhances PMA- and IL-1 $\beta$-induced COX-2 expression in HEI-OCl cells. PMA and IL-1 $\beta$ have previously been demonstrated to induce COX-2 expression (17-23). Western blotting and RT-PCR were performed to determine the effect of rottlerin on PMA- and IL-1 $\beta$-induced COX-2 expression in HEI-OC1 cells. As demonstrated in Fig. 5, treatment with rottlerin enhances PMA and IL-1 $\beta$-induced COX-2 protein and mRNA expression compared with treatment with PMA/IL-1 $\beta$ alone.

\section{Discussion}

To the best of our knowledge, the present study is the first to demonstrate that treatment of HEI-OC1 cells with rottlerin markedly increases COX-2 protein and mRNA expression levels. COX-2 expression is regulated differently depending on the stimulus, cellular environment and cell type (24). Previous mechanistic studies of COX-2 transcriptional regulation have demonstrated that the transcription factors nuclear factor (NF)- $\kappa \mathrm{B}, \mathrm{NF}-\mathrm{IL}-6$, cAMP response element and activator protein-1 are critical for COX-2 expression (24-26). Additionally, post-transcriptional regulation of COX-2 expression has been predominantly attributed to stabilization of the COX-2 mRNA by 38 MAPK signaling and RNA binding proteins (including ELAV-like RNA binding protein 1 and ZFP36 ring finger protein) (27). Studies using pharmacological MAPK inhibitors demonstrated the importance of p38MAPK in rottlerin-induced COX-2 upregulation in HEI-OC1cells.

Previous studies demonstrated that rottlerin induces several characteristic ER stress markers, including upregulation of heat shock protein family A member 5 and DNA damage inducible transcript 3 , phosphorylation of eukaryotic translation initiation factor $2 \alpha$ and ER stress-specific X-box binding protein-1 splicing (28). ER stress-inducing agents enhance COX-2 expression in various cell types $(16,29)$. The present study demonstrated that suppression of ATF4 using siRNA attenuated rottlerin-induced COX-2 expression (Fig. 4B), suggesting that ATF4 may be critical for rottlerin-induced COX-2 expression. Rottlerin enhances IL-1 $\beta$-induced COX-2 expression through sustained p38MAPK activation in breast cancer cells (30). Rottlerin had been previously demonstrated as a specific inhibitor of PKCס (5). However in a previous study, suppression of PKC $\delta$ expression with siRNA did not upregulate IL-1 $\beta$-induced COX-2 expression (30). Furthermore, rottlerin has been demonstrated to induce pro-apoptotic ER stress via the PKC $\delta$-independent pathway (28). Thus, PKC $\delta$ may be not involved in rottlerin- and IL-1 $\beta$-induced COX-2 upregulation in HEI-OC1 cells.

Feng et al (31) reported that reactive oxygen intermediates are a specific and important regulator $\mathrm{COX}-2$ expression induced by IL-1, tumor necrosis factor- $\alpha$ and LPS. A previous study demonstrated that rottlerin increases heme oxygenase-1 (HO-1) expression through 
A

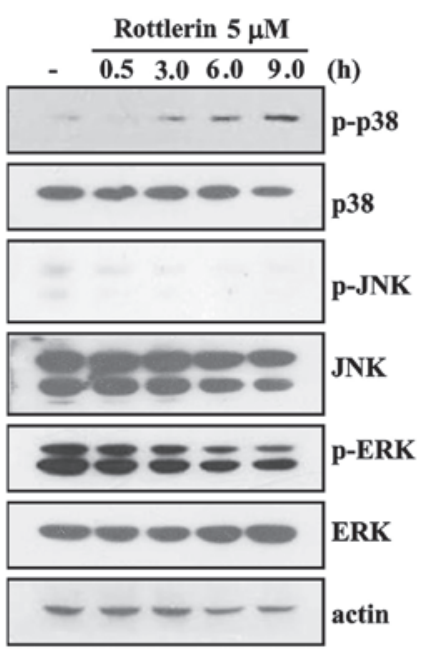

B

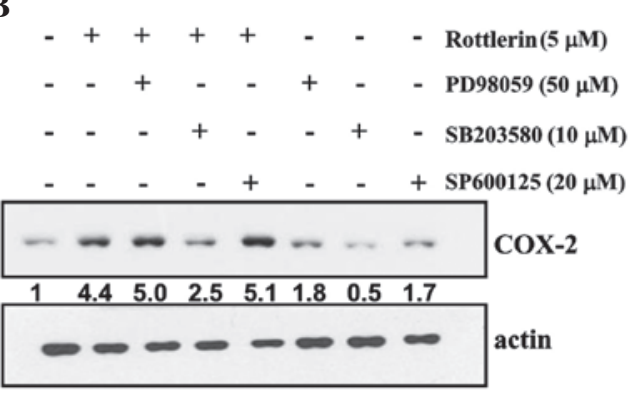

C

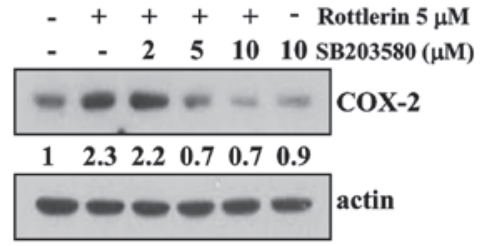

Figure 3. p38MAPK is associated with rottlerin-induced COX-2 expression in HEI-OC1 cells. (A) HEI-OC1 cells were treated with $5 \mu \mathrm{M}$ rottlerin for the indicated time periods. The protein expression levels of phopho (p)-p38 MAPK, p38, p-JNK, JNK, p-ERK, ERK and actin were determined by western blotting. (B) HEI-OC1 cells were pretreated with the ERK inhibitor PD98059 $(50 \mu \mathrm{M})$, the p38MAPK inhibitor SB203580 $(10 \mu \mathrm{M})$ or a JNK inhibitor SP600125 $(20 \mu \mathrm{M})$, and treated with $5 \mu \mathrm{M}$ rottlerin for $12 \mathrm{~h}$. The protein expression levels of COX-2 and actin were determined by western blotting. (C) HEI-OC1 cells were pretreated with the indicated concentrations with SB203580, and treated with $5 \mu \mathrm{M}$ rottlerin for $12 \mathrm{~h}$. The protein expression levels of COX-2 and actin were determined by western blotting. Actin served as a loading control. p-, phospho; p38MAPK, p38 mitogen-activated protein kinase; JNK, c-Jun N-terminal kinase; ERK, extracellular signal-regulated kinase; COX-2, cyclooxygenase-2.

A

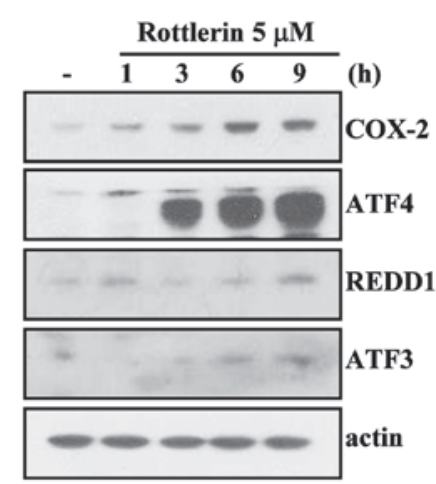

B

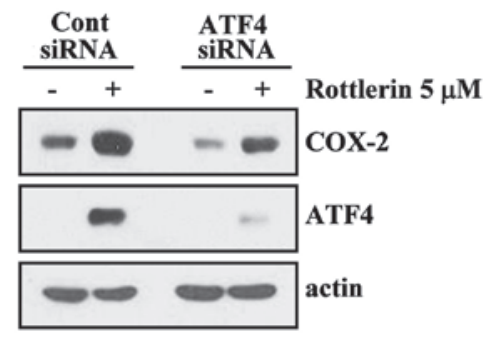

Figure 4. ATF4 is associated with rottlerin-induced COX-2 expression in HEI-OC1 cells. (A) HEI-OC1 cells were treated with $5 \mu \mathrm{M}$ rottlerin for the indicated time periods. The protein expression levels of COX-2, ATF4, REDD1 and ATF3 were determined by western blotting. (B) HEI-OC1 cells were transfected with ATF4 siRNA or control siRNA. Following transfection, cells were treated with or without $5 \mu \mathrm{M}$ rottlerin for $12 \mathrm{~h}$. The protein expression levels of COX-2, ATF4 and actin were determined by western blotting. Actin served as a loading control for western blot analysis. COX-2, cyclooxygenase-2; ATF, activating transcription factor; REDD1, regulated in development and DNA damage responses 1.

ROS generation. Rottlerin-induced HO-1 upregulation was abrogated in the presence of NAC antioxidant (12). However, in the present study, pretreatment with a ROS scavenger did
A

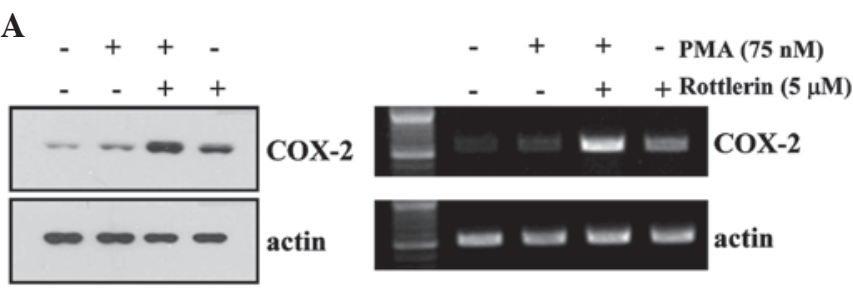

B

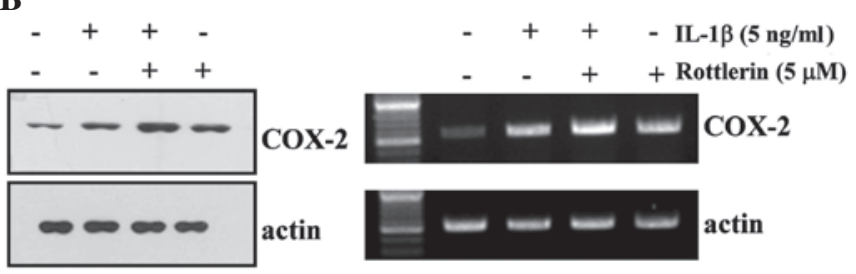

Figure 5. Rottlerin enhances PMA- and IL-1 $\beta$-induced COX-2 expression in HEI-OC1 cells. HEI-OC1 cells were treated with (A) $75 \mathrm{nM}$ PMA or (B) $5 \mathrm{ng} / \mathrm{ml} \mathrm{IL-1 \beta}$ in the absence or presence of $5 \mu \mathrm{M}$ rottlerin for $12 \mathrm{~h}$. The protein and mRNA expression levels of COX-2 and actin were determined by western blotting and reverse transcription-polymerase chain reaction, respectively. Actin served as a loading control. PMA, phorbol-12-myristate-13-acetate; COX-2 cyclooxygenase-2; IL-1 $\beta$, interleukin-1 $\beta$.

not suppress rottlerin-induced COX-2 upregulation. The precise mechanisms underlying ROS-mediated regulation of rottlerin-induced COX-2 upregulation remain to be fully elucidated. Furthermore, COX-2 expression was enhanced when HEI-OC1 cells were concurrently treated with rottlerin and IL- $1 \beta$ or PKC activator, PMA. The findings of the present study suggest that other signaling pathways are involved in rottlerin-induced COX-2 expression in human auditory cells, which require further investigation. Additional research is expected to be beneficial for the treatment of cochlear inflammation-associated hearing loss. 


\section{Acknowledgements}

The present study was supported by an NRF grant funded by the Korean Government (MSIP; grant no. 2014R1A5A2010008) and a 2015 Scholar Research Grant from Keimyung University (Daegu, South Korea).

\section{References}

1. Smith WL, DeWitt DL and Garavito RM: Cyclooxygenases: Structural, cellular, and molecular biology. Annu Rev Biochem 69: 145-182, 2000.

2. Tsatsanis C, Androulidaki A, Venihaki M and Margioris AN: Signalling networks regulating cyclooxygenase-2. Int J Biochem Cell Biol 38: 1654-1661, 2006.

3. Griswold DE and Adams JL: Constitutive cyclooxygenase (COX-1) and inducible cyclooxygenase (COX-2): Rationale for selective inhibition and progress to date. Med Res Rev 16: 181-206, 1996.

4. Tanigawa T, Odkhuu E, Morikawa A, Hayashi K, Sato T, Shibata R, Goto F, Ueda H and Yokochi T: Immunological role of prostaglandin $\mathrm{E} 2$ production in mouse auditory cells in response to LPS. Innate Immun 20: 639-646, 2014.

5. Gschwendt M, Müller HJ, Kielbassa K, Zang R, Kittstein W, Rincke $\mathrm{G}$ and Marks F: Rottlerin, a novel protein kinase inhibitor. Biochem Biophys Res Commun 199: 93-98, 1994.

6. Cross T, Griffiths G, Deacon E, Sallis R, Gough M, Watters D and Lord JM: PKC-delta is an apoptotic lamin kinase. Oncogene 9: 2331-2337, 2000.

7. Kontny E, Kurowska M, Szczepańska K and Maśliński W: Rottlerin, a PKC isozyme-selective inhibitor, affects signaling events and cytokine production in human monocytes. J Leukoc Biol 67: 249-258, 2000.

8. Hsieh HL, Wang HH, Wu CY, Jou MJ, Yen MH, Parker P and Yang CM: BK-induced COX-2 expression via PKC-delta-dependent activation of $\mathrm{p} 42 / \mathrm{p} 44 \mathrm{MAPK}$ and NF-kappaB in astrocytes. Cell Signal 19: 330-340, 2007.

9. Soltoff SP: Rottlerin: An inappropriate and ineffective inhibitor of PKCdelta. Trends Pharmacol Sci 8: 453-458, 2007

10. Song KS, Kim JS, Yun EJ, Kim YR, Seo KS, Park JH, Jung YJ, Park JI, Kweon GR, Yoon WH, et al: Rottlerin induces autophagy and apoptotic cell death through a PKC-delta-independent pathway in HT1080 human fibrosarcoma cells: The protective role of autophagy in apoptosis. Autophagy 4: 650-658, 2008.

11. Lim JH, Park JW, Choi KS, Park YB and Kwon TK: Rottlerin induces apoptosis via death receptor 5 (DR5) upregulation through CHOP-dependent and PKC delta-independent mechanism in human malignant tumor cells. Carcinogenesis 30: 729-736, 2009.

12. Park EJ, Lim JH, Nam SI, Park JW and Kwon TK: Rottlerin induces heme oxygenase-1 (HO-1) up-regulation through reactive oxygen species (ROS) dependent and PKC delta-independent pathway in human colon cancer HT29 cells. Biochimie 92: 110-115, 2010.

13. LeBel CP, Ischiropoulos $\mathrm{H}$ and Bondy SC: Evaluation of the probe $2{ }^{\prime}, 7^{\prime}$-dichlorofluorescin as an indicator of reactive oxygen species formation and oxidative stress. Chem Res Toxicol 5: 227-231, 1992.

14. Lu Y and Wahl LM: Oxidative stress augments the production of matrix metalloproteinase-1, cyclooxygenase-2, and prostaglandin E2 through enhancement of NF-kappa B activity in lipopolysaccharide-activated human primary monocytes. J Immunol 175: 5423-5429, 2005.

15. Chen JJ, Huang WC and Chen CC: Transcriptional regulation of cyclooxygenase-2 in response to proteasome inhibitors involves reactive oxygen species-mediated signaling pathway and recruitment of CCAAT/enhancer-binding protein delta and CREB-binding protein. Mol Biol Cell 16: 5579-5591, 2005 .
16. Hung JH, Su IJ, Lei HY, Wang HC, Lin WC, Chang WT, Huang W, Chang WC, Chang YS, Chen CC and Lai MD: Endoplasmic reticulum stress stimulates the expression of cyclooxygenase-2 through activation of NF-kappaB and pp38 mitogen-activated protein kinase. J Biol Chem 279: 46384-46392, 2004.

17. Huang ZF, Massey JB and Via DP: Differential regulation of cyclooxygenase-2 (COX-2) mRNA stability by interleukin-1 beta (IL-1 beta) and tumor necrosis factor-alpha (TNF-alpha) in human in vitro differentiated macrophages. Biochem Pharmacol 59: 187-194, 2000.

18. Nakao S, Ogtata Y, Shimizu E, Yamazaki M, Furuyama S and Sugiya H: Tumor necrosis factor alpha (TNF-alpha)-induced prostaglandin $\mathrm{E} 2$ release is mediated by the activation of cyclooxygenase-2 (COX-2) transcription via NFkappaB in human gingival fibroblasts. Mol Cell Biochem 238: 11-18, 2002.

19. Molina-Holgado E, Ortiz S, Molina-Holgado F and Guaza C: Induction of COX-2 and PGE(2) biosynthesis by IL-1beta is mediated by PKC and mitogen-activated protein kinases in murine astrocytes. Br J Pharmacol 131: 152-159, 2000.

20. Miralpeix M, Camacho M, López-Belmonte J, Canalías F, Beleta J, Palacios JM and Vila L: Selective induction of cyclo-oxygenase-2 activity in the permanent human endothelial cell line HUV-EC-C: Biochemical and pharmacological characterization. Br J Pharmacol 121: 171-180, 1997.

21. Shishodia S, Koul D and Aggarwal BB: Cyclooxygenase (COX)-2 inhibitor celecoxib abrogates TNF-induced NF-kappa B activation through inhibition of activation of I kappa B alpha kinase and Akt in human non-small cell lung carcinoma: Correlation with suppression of COX-2 synthesis. J Immunol 73: 2011-2022, 2004.

22. Mitchell JA, Belvisi MG, Akarasereenont P, Robbins RA, Kwon OJ, Croxtall J, Barnes PJ and Vane JR: Induction of cyclo-oxygenase-2 by cytokines in human pulmonary epithelial cells: Regulation by dexamethasone. Br J Pharmacol 113: 1008-1014, 1994

23. Jiang YJ, Lu B, Choy PC and Hatch GM: Regulation of cytosolic phospholipase A2, cyclooxygenase-1 and -2 expression by PMA, TNFalpha, LPS and M-CSF in human monocytes and macrophages. Mol Cell Biochem 246: 31-38, 2003.

24. Kang YJ, Mbonye UR, DeLong CJ, Wada M and Smith WL: Regulation of intracellular cyclooxygenase levels by gene transcription and protein degradation. Prog Lipid Res 46: 108-125, 2007.

25. Tamura M, Sebastian S, Yang S, Gurates B, Fang Z, Okamura K and Bulun SE: Induction of cyclooxygenase-2 in human endometrial stromal cells by malignant endometrial epithelial cells: Evidence for the involvement of extracellularly regulated kinases and CCAAT/enhancer binding proteins. J Mol Endocrinol 31: 95-104, 2003.

26. Wardlaw SA, Zhang $\mathrm{N}$ and Belinsky SA: Transcriptional regulation of basal cyclooxygenase- 2 expression in murine lung tumor-derived cell lines by CCAAT/enhancer-binding protein and activating transcription factor/cAMP response element-binding protein. Mol Pharmacol 62: 326-333, 2002.

27. Lasa M, Mahtani KR, Finch A, Brewer G, Saklatvala J and Clark AR: Regulation of cyclooxygenase 2 mRNA stability by the mitogen-activated protein kinase p38 signaling cascade. Mol Cell Biol 20: 4265-4274, 2000.

28. Lim JH, Park JW, Kim SH, Choi YH, Choi KS and Kwon TK: Rottlerin induces pro-apoptotic endoplasmic reticulum stress through the protein kinase C-delta-independent pathway in human colon cancer cells. Apoptosis 13: 1378-1385, 2008.

29. Rasheed $Z$ and Haqqi TM: Endoplasmic reticulum stress induces the expression of COX-2 through activation of eIF2 $\alpha$, p38-MAPK and NF- $\kappa$ B in advanced glycation end products stimulated human chondrocytes. Biochim Biophys Acta 1823: 2179-2189, 2012.

30. Park EJ and Kwon TK: Rottlerin enhances IL-1 $\beta$-induced COX-2 expression through sustained p38 MAPK activation in MDA-MB-231 human breast cancer cells. Exp Mol Med 43: 669-675, 2011.

31. Feng L, Xia Y, Garcia GE, Hwang D and Wilson CB: Involvement of reactive oxygen intermediates in cyclooxygenase- 2 expression induced by interleukin-1, tumor necrosis factor-alpha, and lipopolysaccharide. J Clin Invest 95: 1669-1675, 1995. 